\title{
A Generalized Model for Cost and Fairness Analysis in Coded Cooperative Data Exchange
}

\author{
Shahriar Etemadi Tajbakhsh, Parastoo Sadeghi and Ramtin Shams \\ Research School of Information Sciences and Engineering \\ The Australian National University \\ Canberra, 0200, Australia \\ Emails: $\{$ shahriar.etemadi-tajbakhsh, parastoo.sadeghi,ramtin.shams\}@anu.edu.au
}

\begin{abstract}
We consider the issues of cost and fairness in the problem of cooperative data exchange among a group of wireless clients. In this problem, each client initially holds a subset of packets and needs to obtain the full set of packets through cooperation with other clients via a shared broadcast channel. To find minimum cost transmission schemes, we propose a general model for the problem which is based on network information flow with side information available to the sinks. As a special case of minimum cost solutions, the minimum number of required transmissions is studied in detail. We show that packet splitting is a natural consequence of solving the linear programming associated with the mentioned network flow problem. Our main observation is that splitting the packets not only minimizes the number of transmissions, but also it results in considerably more fairness compared to the case where splitting is not allowed. Hence, incentive-based long-term cooperation among users can be sustained.
\end{abstract}

Keywords- network coding, cooperative data exchange, linear programming, multicast, side information.

\section{INTRODUCTION}

\section{A. Motivation}

Cooperation among users of a wireless network is known to be advantageous in terms of freeing valuable server resources and enabling network scalability [1]. For establishing longterm and reliable user interactions, it is important to consider their cost and also to provide incentives for cooperation. The ability to achieve maximum fairness (usually in terms of individual user energy or bandwidth) and minimum cost (usually total energy or bandwidth) can determine whether a cooperative protocol can be successfully established and maintained or not.

In this paper, we analyze the issues of cost and fairness in a recently-introduced problem, referred to as coded cooperative data exchange [2]. In this problem, a group of closely-located wireless clients are interested in downloading a set of packets, and initially each client holds a subset of packets. The clients wish to exchange (possibly network coded packets) through a common broadcast channel to obtain all the packets. This problem can have a variety of applications and advantages in cellular systems and other server-based wireless broadcast networks with an ever increasing demand for better download quality and higher data rates. For example, due to the occurrence of packet erasures over a wireless channel, a client might not receive the entire set of packets from the server. This motivates one to use the short range links among the nearby clients which are faster, cheaper, and more reliable instead of exhaustive retransmissions by the base station, wasting its valuable downlink bandwidth.

\section{B. Related Work and Knowledge Gaps}

Cooperative data exchange has been the subject of recent research, where numerous works have studied different aspects of the problem (note that different names are used to refer to essentially the same problem). Some heuristic and randomized algorithms have been proposed to minimize the delay [3], [4] or the total number of transmissions [2], [5]. However, finding the exact optimal solution has proven to be non-trivial and a deeper understanding of the problem, how to best model it, and its properties is still required.

Recently, it has been shown independently in [6], [7] that linear programming can be used to guarantee that each client will receive sufficient number of packets, while the total number of transmissions is minimized. It is found that the solution to linear programming often requires the packets to be split into so-called subpackets. This improved the findings of [2], [5] which did not allow or consider packet splitting. However, neither [7] nor [3], [4], [2], [5] studied the issue of fairness in terms of number of individual transmissions of different users. Moreover, the modeling and formulation of linear programming in [6], [7] is only suited when the cost is defined as the total number of transmissions and cannot incorporate more general cost functions. The problem of minimum cost cooperative data exchange has been recently studied in [8], but the adopted approach is a generalization of the randomized algorithm of [5], which is not model-based and does not consider packet splitting.

To the best of our knowledge, a generalized network model for cooperative data exchange that is easy to understand and provides a general framework for the joint analysis of cost and fairness is still missing in the literature. This is the topic of this paper. Before summarizing our contributions, we briefly discuss an example to clarify the impact of packet splitting on cost and fairness.

\section{An Example of Simultaneous Cost Reduction and Fairness Improvement}

Consider the example shown in Fig. 1(a). Three wireless clients, $c_{1}, c_{2}$ and $c_{3}$ hold packets $\left\{x_{1}, x_{2}\right\},\left\{x_{1}, x_{3}\right\}$ and 


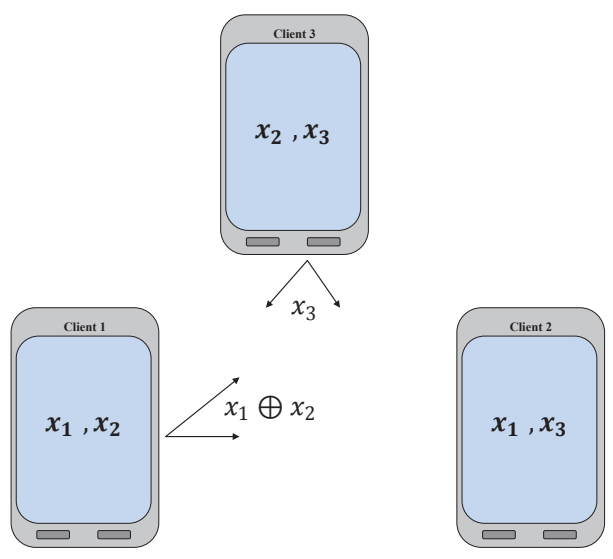

(a)

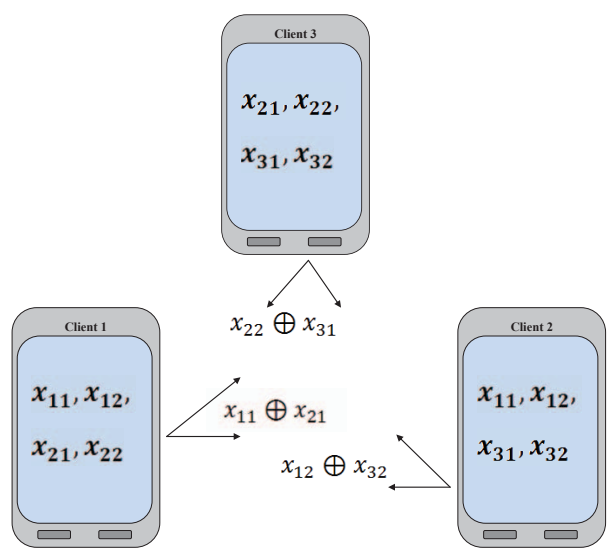

(b)

Fig. 1. (a) Cooperative data exchange without packet splitting, (b) Cooperative data exchange with packet splitting.

$\left\{x_{2}, x_{3}\right\}$, respectively where packets are elements from a finite field, i.e. $x_{1}, x_{2}, x_{3} \in G F\left(2^{m}\right)$. Using linear network coding [9], it has been shown in [2] that all clients can obtain their missing packets by 2 transmissions as shown in Fig. 1(a), thus reducing the transmissions compared to an uncoded scheme by $33 \%$. Note that in this solution, $c_{2}$ does not transmit and only benefits from other users' transmissions. So the scheme is not fair.

Here, the implicit assumption is that the packets are indivisible units of information, but if we allow the clients to split the packets to smaller elements, we may achieve better solutions, or more technically, a solution which is not only optimal but fairer than a solution without splitting. To clarify, suppose that $x_{1}, x_{2}$ and $x_{3}$ are now divided to subpackets $\left\{x_{11}, x_{12}\right\},\left\{x_{21}, x_{22}\right\}$ and $\left\{x_{31}, x_{32}\right\}$, respectively, as shown in Fig. 1(b). It is easy to verify that if $c_{1}$ broadcasts $x_{11} \oplus x_{21}$, $c_{2}$ broadcasts $x_{12} \oplus x_{32}$, and $c_{3}$ broadcasts $x_{22} \oplus x_{31}$, then all the clients can obtain what they need. Compared to [2], this further reduces the total number of transmissions from 2 full packets to 3 half-packets or simply 1.5 packets. Moreover, everyone participates equally in the exchange and full fairness is achieved.

\section{Summary of Contributions}

Below we summarize our main contributions and highlight their distinctions with previous works.

- In Section II-B, we show that the cooperative data exchange is a special class of multicast network information flow problems with available side information at the sinks [10]. This leads us to conclude that linear network coding is sufficient to achieve optimal solutions for cooperative data exchange.

- In Section II-C, we model and formulate this problem as a minimum cost subgraph given a linear cost criterion leading to linear programming. By defining an appropriate cost function we can solve the problem for the minimum number of transmissions. However, the model in its general form provides the capability to control different performance metrics and/or to model the problem in more realistic conditions as discussed in II-C.

- In Sections III and IV, we apply a normalized fairness index to the solution of linear programming to show that splitting the packets results in a significant improvement in fairness.

- In Section IV, we show through numerical simulations that while packet splitting can reduce the total number of transmissions, the average improvement compared to integral packet coding is negligible, while the improvement in fairness is considerable.

Since our proposed model is based on the network information flow with side information, it is easy to understand and study. Moreover, our model is more general than that studied in [7] which specialized on the minimum number of transmissions as the cost function. Compared to [8], it naturally allows model-based packet splitting while minimizing the cost. Finally, the solutions to the data exchange problem are tested for fairness among users, which to the best of our knowledge, has not been considered in the literature before.

\section{Optimal SOLUTION}

\section{A. Problem Definition}

The cooperative data exchange problem is defined formally as follows. A set $X=\left\{x_{1}, \ldots, x_{n}\right\}$ of $n$ packets needs to be delivered to a set of $k$ clients $C=\left\{c_{1}, \ldots, c_{k}\right\}$. Initially, each client knows a subset of packets denoted by $\Omega_{i} \subseteq X$, while the clients collectively know all the packets in $X$, i.e., $\bigcup_{c_{i} \in C} \Omega_{i}=X$. The objective of each client is to obtain its missing packets $\bar{\Omega}_{i}=X \backslash \Omega_{i}$ with the help of its peers. The problem is to find the minimum cost transmission scheme assuming the clients can broadcast coded packets via an erasure-free channel and each client is aware of the initial distribution of packets. Similar assumptions have been made in [2], [4], [7], [8], which help understand intrinsic aspects of the problem. Unlike [2], [5], we allow the packets to be split to some subpackets if necessary. More precisely, a packet $x_{i}$ of length $m_{i}$ might be partitioned to a set of $l$ subpackets $\left\{x_{i, 1}, \ldots, x_{i, l}\right\}$, such that each subpacket $x_{i, d}$ has a length $\mu_{i, d} \leq m_{i}$ and $\sum_{d} \mu_{i, d}=m_{i}$. 
Based on the concept of subpackets, with linear network coding, at each transmission round $t$ a client $c_{i}$ may transmit a linear combination of the subpackets

$$
p_{t}=\sum_{x_{j, d} \in \Omega_{i}^{*}} \lambda_{t}^{j, d} x_{j, d}
$$

where $\Omega_{i}^{*}$ is the set of all subpackets client $c_{i}$ holds and $\lambda_{t}^{j, d}$, $\mathrm{s}$ are the network coding coefficients. Whether packets should be split and by how much depends on the solution found by the linear programming, which will be discussed in the next two subsections.

\section{B. Graph Model}

In this paper, we study the cooperative data exchange as a network information flow problem. Based on the observation in the example of Fig. 1(b) and other examples, it is concluded that achieving the optimal solution may not be possible without breaking the packets to smaller so-called subpackets. This fact motivated us to investigate the optimal number of transmissions (or more generally minimum cost solutions) in this problem through a network information flow model. At first, we show how this problem is modeled as a layered multicast network with side information available at the sinks. Then the optimal solution is found using a linear programming formulation.

We form an equivalent acyclic graph $G(V, E)$ of the data exchange problem as follows. $V$ is the set of vertices which includes five types of nodes:

- The set of sources $S=\left\{x_{1}, \ldots, x_{n}\right\}$, i.e. each packet $x_{j}$ can be considered as a source node. We treat each packet $x_{j}$ as a realization of a random process $X_{j}$, where the corresponding random processes are mutually independent. We denote the set of all random processes generated at the sources by $\mathcal{X}=\left\{X_{1}, \ldots, X_{n}\right\}$.

- A virtual source $s^{*}$ which provides all the random processes in $\mathcal{X}$.

- The set of encoders $U=\left\{u_{1}, \ldots, u_{k}\right\}$. Since any client is able to generate a combination of the packets in its possession, it can be considered as an encoder.

- The set of virtual nodes $U^{\prime}=\left\{u_{1}^{\prime}, \ldots, u_{k}^{\prime}\right\}$, where each encoder has a corresponding virtual node to which it broadcasts the encoded packets it generates.

- The set of sinks (decoders) $T=\left\{t_{1}, \ldots, t_{k}\right\}$. A client can simultaneously play the role of an encoder and a decoder. Based on the definition of our problem, each client $c_{i}$ holds a subset of packets which can be considered as a realization of a set of random processes denoted by $\mathcal{Y}_{i} \subseteq \mathcal{X}$.

Therefore, $V=S \cup\left\{s^{*}\right\} \cup U \cup U^{\prime} \cup T . E \subseteq(V \times V)$ is the set of noiseless edges, which is divided into the following five types:

- The set of edges connecting the virtual source $s^{*}$ to the set of sources, which is denoted by $\mathcal{E}_{1}=\left\{\left(s^{*}, v_{j}\right) \mid v_{j} \in S\right\}$.

- The set of edges connecting the sources to the nodes in $U$ based on the initial distribution of packets, $\mathcal{E}_{2}=$ $\left\{\left(v_{i}, v_{j}\right) \mid v_{i} \in S, v_{j} \in U, x_{i} \in \Omega_{j}\right\}$.

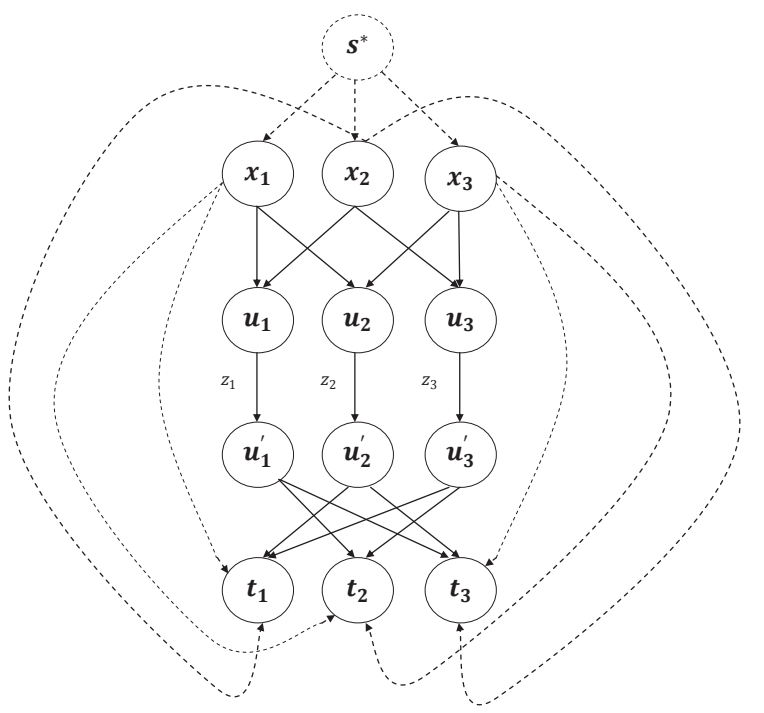

Fig. 2. The equivalent graph of the example in Fig. 1

- The set of edges connecting a node in $U$ to its corresponding node in $U^{\prime}, \mathcal{E}_{3}=\left\{\left(u_{i}, u_{i}^{\prime}\right) \mid u_{i} \in U, u_{i}^{\prime} \in U^{\prime}\right\}$. This guarantees that whenever a client generates a function of the packets in its possession, it is naturally broadcast to the intended sinks through the corresponding node in $U^{\prime}$.

- The set of edges which broadcast the coded packets to all the neighbors, i.e., $\mathcal{E}_{4}=\left\{\left(u_{i}^{\prime}, t_{j}\right) \mid u_{i}^{\prime} \in U^{\prime}, t_{j} \in T, j \neq\right.$ $i\}$.

- The set of edges providing the side information to the sinks, i.e., $\mathcal{E}_{5}=\left\{\left(v_{i}, t_{j}\right) \mid v_{i} \in S, x_{i} \in \Omega_{j}\right\}$.

We also denote the incoming and outgoing edges of a node $v$ by $\Gamma_{i}(v), \Gamma_{o}(v)$ respectively. Fig. 2 shows the equivalent graph of the example in Fig. 1.

We remark that our proposed graph model is jointly inspired by the networks proposed and studied in [10], [11]. In [11], a system of distributed source coding for satellite communications was modeled and a layered network was constructed to represent transmitters, encoders and decoders (at receivers). Each transmitter can send information to a subset of encoders and each decoder has access to a subset of encoders. Our model possesses a layered encoder/decoder topology similar to [11] and combines it with the concept of multicast with side information [10] available at the sinks. For such a network, it is shown that linear network codes are able to achieve its rate region and the rate region is simply an extension of cutset bounds based on the available side information [10]. Since our model inherits the properties of [10] and [11], we conclude that linear network codes are sufficient for achieving optimal solution to cooperative data exchange found by linear programming. This is outlined in the subsection below.

\section{Linear Programming Framework}

To find the minimum cost transmission scheme, we apply linear programming to its corresponding graph. This is similar 
to the minimum cost subgraph calculation in [12], with two main differences: (1) In our problem, each sink holds a set of packets as side information which is provided by the edge set $\mathcal{E}_{5}$ at no cost; (2) We only need to associate actual rates $z_{i}$ to the edges in $\mathcal{E}_{3}$ for $i=1, \ldots, k$ to take the number of actual transmissions into account. We also associate a set of variables to each edge denoted by $\alpha_{e}^{t_{i}}$, which can be considered as the flow towards the sink $t_{i}$ carried by the edge $e \in E$. In its most general form, the corresponding linear programming can be formulated in the following way:

$$
\begin{gathered}
\sum_{i=1}^{k} b_{i} z_{i} \\
\text { minimizect to } \\
0 \leq \alpha_{e}^{t_{i}} \leq z_{i} \leq C_{i} \\
\sum_{\Gamma_{o}(v)} \alpha_{e}^{t_{i}}-\sum_{\Gamma_{i}(v)} \alpha_{e}^{t_{i}}=\sigma_{v}^{\left(t_{i}\right)}
\end{gathered}
$$

where,

$$
\sigma_{v}^{\left(t_{i}\right)}= \begin{cases}R, & \text { if } v=s^{*}, \\ -R, & \text { if } v=t_{i}, \\ 0, & \text { otherwise. }\end{cases}
$$

where $b_{i}$ is the cost associated with a transmission by client $c_{i}, C_{i}$ is the capacity of the $i$ th edge in $\mathcal{E}_{3}$, and $R=\sum_{j=1}^{n} m_{j}$ where $m_{j}$ is the length of packet $x_{j}$. It should be noted that we set $\alpha_{e}^{t_{i}}=m_{j}$ for $e=\left(s^{*}, v_{j}\right)$. This enables us to solve the problem even in the case that network packets do not have the same length.

Modeling cooperative data exchange in the way discussed here, not only gives a deep understanding about the nature of the problem, but also it provides a powerful tool to adjust the performance metrics of the network. For example, we may limit the total number of transmissions by each client based on its available battery or just to maintain more fairness among the clients. Also, the ability of handling packets of different lengths, might be helpful to extend the idea of partial packet recovery suggested by [13] to such cooperative settings. Each client might have heard a packet partially, so instead of discarding the incomplete packet it can exploit its partial knowledge of that packet in cooperation with its peers. Moreover, short range links may have different qualities, that can be taken into account in the associated costs or can limit the capacity of actual transmission links.

Clearly, finding the minimum number of required transmissions is a special case of the minimum cost problem. This can be achieved if we set $b_{i}=1$ and $C_{i}=\infty$ for all $i=1, \ldots, k$. To count the number of transmissions in terms of packets, we can set $R=n$ and $\alpha_{e}^{t_{i}}=1$. For instance, in the example of Fig. 2, solving the linear programming would result in $z_{1}=z_{2}=z_{3}=1 / 2$. It is assumed that the size of packets is appropriate to be divided if needed. In this example, $m_{j}$ 's are assumed to be equal and even numbers. However, when the size of packets is not even, there would still be some benefit in packet splitting in an asymmetric fashion, which is not discussed here any further.

\section{FAIRNESS}

Unlike the conventional communication systems where users compete against each other to capture the resources of the network, in cooperative models, the clients aid each other to achieve a common goal. Cooperation may result in a dramatic improvement in the usage of network resources. However, a fundamental question arises: under what conditions users are motivated to cooperate? In other words, not only the efficiency of the whole system should be considered, but each user, individually should find a major benefit in participating. From this point of view, in a cooperative system, the allocation of resources and duties should be as fair as possible while a certain set of constraints and optimality conditions are guaranteed. It is important to note that the concept of fairness in a cooperative system is not equivalent to uniform distribution of resources and tasks, because a 'totally fair' system with an overall poor performance is not desired even from the users' perspective.

As it was mentioned earlier, splitting the packets may result in more fairness, i.e. the portion of transmissions by each client is close to the others as much as possible. Roughly speaking, by splitting the packets we enable more clients to participate in the transmission of a single packet. In our problem, fairness is a critical issue, since the energy supply of wireless transceiver devices is usually limited.

In the following, we will have a brief introduction to a statistical index of fairness and its advantages over other metrics and its application to our problem. To have a quantitative measure of fairness, different schemes and formulas might be considered. For example, at a first look, the variance of $z_{i}$ 's found in (1) seems to be a good candidate. Lower variance may indicate more fairness around a specific mean, but the most important weakness of variance is its dependence on the mean and the size of the problem. [14] discusses the desired properties of a fairness index and suggests a very useful statistical formula to measure the fairness, which will be used in this paper. Using the notations in this paper, the index proposed by [14] can be written as

$$
f(\mathbf{z})=\frac{\left(\sum_{i=1}^{k} z_{i}\right)^{2}}{k \sum_{i=1}^{k} z_{i}^{2}}
$$

where $\mathbf{z}=\left(z_{1}, z_{2}, \cdots, z_{k}\right)$ is the vector of $z_{i}$ 's. This index is a normalized measure which is independent of the scales, population and size of the problem, and continuous. For more details see [14]. Applying this formula to the canonic example of Fig. 1, gives $f(\mathbf{z})=\frac{2}{3}$ for the non-splitting case where $\mathbf{z}=(1,1,0)$ and $f(\mathbf{z})=1$ for the splitting case where $\mathbf{z}=\left(\frac{1}{2}, \frac{1}{2}, \frac{1}{2}\right)$.

As long as the optimal solution is unique, exploring the fairest solution is not meaningful, because any other solution with more fairness would be suboptimal in terms of number of transmissions (although clients may come to an agreement to sacrifice some optimality in the total number of transmissions in favor of a fairer solution). But in the case that the optimal solution is not unique, it is reasonable to choose the fairest 
optimal solution. The uniqueness of solution to a linear optimization problem can be determined before solving the problem using the method in [15]. In fact, we have observed through simulations that in a large number of instances of our problem, the solution is not unique. We denote the total number of transmissions in an optimal solution found by the LP by $z^{*}$. To find the fairest optimal solution, we should maximize the nonlinear fairness index subject to the same set of conditions used by the LP, given that the summation of $z_{i}$ 's is equal to $z^{*}$. That is,

$$
\begin{aligned}
\operatorname{maximize} \quad f\left(\mathbf{z}^{\prime}\right) & =\frac{\left(\sum_{i=1}^{k} z_{i}^{\prime}\right)^{2}}{k \sum_{i=1}^{k} z_{i}^{\prime 2}} \\
\text { subject to } & \\
\sum_{i=1}^{k} z_{i}^{\prime} & =z^{*} \text { and the set of conditions in (1) }
\end{aligned}
$$

Fortunately, this problem can be converted to a convex minimization problem. By substitution of the condition $\sum_{i=1}^{k} z_{i}^{\prime}=$ $z^{*}$ in the above, the objective function would be become

$$
f\left(\mathbf{z}^{\prime}\right)=\frac{\left(z^{*}\right)^{2}}{k \sum_{i=1}^{k} z_{i}^{\prime 2}}
$$

Since $z^{*}$ is a known constant at this step, this problem is equivalent to the minimization of $\sum_{i=1}^{k} z_{i}^{\prime 2}$. Hence, the problem is converted to a constrained quadratic problem [16].

\section{NumERICAL RESULTS}

In this section we compare the performance of the suggested linear programming in (1) against the algorithm in [5], which is known to be optimal with a high probability if the coding coefficients are chosen from a sufficiently large field under the condition that no packet splitting is allowed. The general structure of the algorithm in [5] is in a way that at each round of transmission, a client with highest rank of knowledge space is selected as the leader to broadcast a linear combination of the packets it holds. In the first part of our simulations, we assumed the cost of transmissions are equal, i.e. the minimum number of required transmissions has been considered.

To generate an initial setting for a cooperative data exchange scenario, we have assumed that each client have received a packet with a probability $p=0.5$. The experiments are performed for $k=8$ clients and different number of packets ranging from $n=4$ to $n=30$ with 250 trials for any value of $n$. Table. I shows the gain of network coding for the two mentioned algorithms over uncoded transmissions, i.e., the ratio of the number of uncoded transmissions to the number of transmissions found by LP or the algorithm in [5]. Although we proved that the optimal solution might not be achievable without splitting the packets, it can be seen from this table that, on average, the gains are very close to each other in practice. But the key advantage of breaking the packets to smaller entities (subpackets) is to improve fairness substantially. Especially, the algorithm in [5] requires nodes
TABLE I

CODING GAIN FOR THE LP AND THE ALGORITHM IN [5].

\begin{tabular}{|l|c|c|c|c|c|c|}
\hline$n$ & 5 & 10 & 15 & 20 & 25 & 30 \\
\hline Alg. [5] & 1.2454 & 1.3867 & 1.4453 & 1.5127 & 1.5461 & 1.5870 \\
\hline LP & 1.2477 & 1.3882 & 1.4485 & 1.5155 & 1.5487 & 1.5886 \\
\hline
\end{tabular}

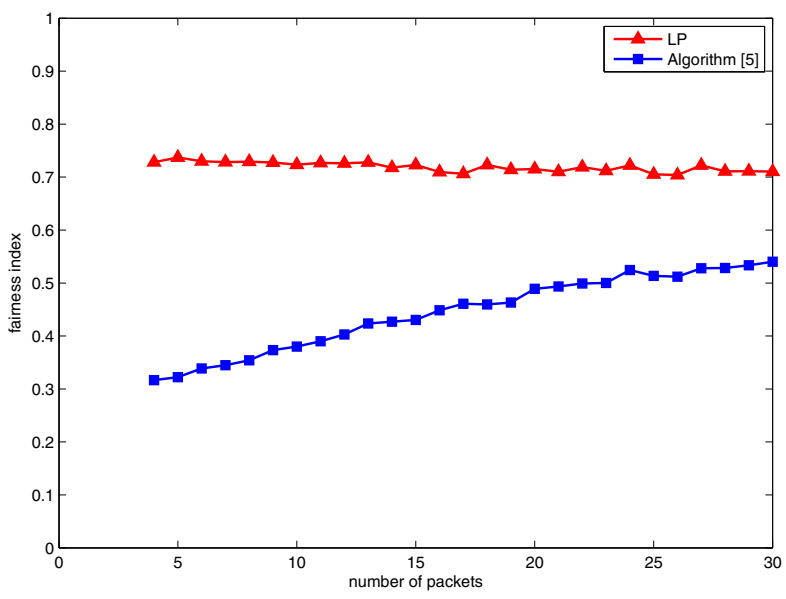

Fig. 3. A comparison between the fairness of LP and the algorithm in [5]

with the higher dimensions of knowledge space to participate more in transmissions, which can be unfair. Fig. 3 shows the fairness index introduced in Section III for the two algorithms. A large gap between the two algorithms can be seen from the fairness point of view. As discussed earlier, splitting the packets provides opportunities for more clients to participate in the transmission of a specific packet. But the gap is narrowed as the number of packets increases. The intuition behind this phenomenon is clear: For larger number of packets, the chance of each client for participating in transmissions is increased in the algorithm of [5].

In the second part of our numerical experiments, we considered the effect of non-uniform costs, where each transmission by client $c_{i}$ has an associated cost $b_{i} \neq 1$. In this experiment we chose $b_{i}$ 's randomly from 1 to 10 for $k=6$ clients and different number of packets ranging from $n=4$ to $n=30$. The results of this experiment has been compared to uncoded transmissions in Fig. 4. Clearly, setting $b_{i}=1$ for all the clients, provides the lower bound for the total number of required transmissions as shown in Fig. 4.

\section{CONClusion}

In this paper, we studied the problem of cooperative data exchange among a group of closely-located wireless clients using network coding. In this problem, each client holds a subset of packets and is interested in achieving all other packets held by other clients through cooperation. We modeled this problem as a multicast with side information available at the sinks to show linear network codes are sufficient to achieve the minimum cost transmission schemes. We introduced a linear program to find the optimal solution, which may naturally result in 


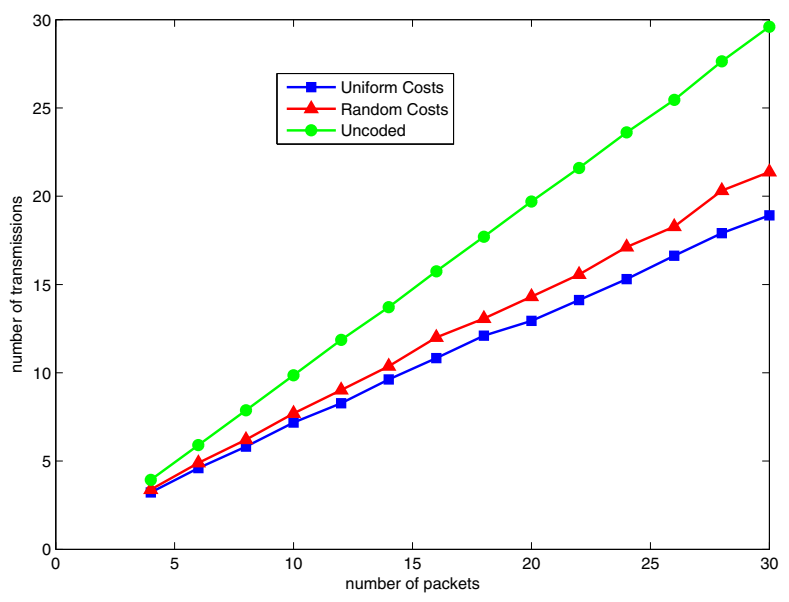

Fig. 4. Number of transmissions for random costs, uniform costs and uncoded transmissions.

fractional numbers for the number of transmissions by each client, which can be interpreted as splitting the packets.

While, on average, splitting the packets only marginally improves the total number of transmissions compared to previously known integral solutions to this problem, it provides a major advantage: a dramatic improvement in fairness. Fairness measures the equity of contributions from individual clients towards the data exchange problem. Obviously, a fairer solution would provide more incentive for clients to cooperate and hence, is a very desirable property of a solution.

In this paper, we assumed that all the clients are fully connected via an erasure-free broadcast channel. This assumption might be relaxed in the future, which indeed adds another non-trivial dimension to the problem and deserves further investigation. Generally, the clients might have a more dynamic behavior (for example, entering and exiting the system during the cooperation phase). Finally, the side information processes at the sinks might have a more general form than just being a subset of packets, which is a scenario that may appear in sensor networks.

\section{ACKNOWLEDGMENT}

This work was supported under Australian Research Council Discovery Projects funding scheme (project no. DP0984950). Authors also would like to acknowledge a helpful discussion with Iman Shames.

\section{REFERENCES}

[1] F. Fitzek and M. Katz, Cooperation in Wireless Communications: Principles and Applications. Springer, 2007.

[2] S. E. Rouayheb, A. Sprinston, and P. Sadeghi, "On coding for cooperative data exchange," in Proc. IEEE Inf. Theory Workshop (ITW), Cairo, Egypt, Jan. 2010, pp. 118-122.

[3] S. Raza, D. Li, C. N. Chuah, and G. Cheung, "Cooperative peer-topeer repair for wireless multimedia broadcast," in IEEE ICME, Beijing, China, July. 2007, pp. 1075-1078.
[4] X. Liu, S. Raza, C. N. Chuah, and G. Cheung, "Network coding based cooperative peer-to-peer repair in wireless ad-hoc networks," in IEEE International Conference of Communication (ICC), Beijing, China, May. 2008, pp. $2153-2158$

[5] A. Sprinston, P. Sadeghi, G. Booker, and S. E. Rouayheb, "A randomized algorithm and performance bounds for coded cooeprative data exchange," in Proc. IEEE Int. Symp. Inf. Theory (ISIT), Austin, TX, Jun. 2010 , pp. $1888-1892$.

[6] S. E. Tajbakhsh, P. Sadeghi, and R. Shams, "The difference between apples and network packets," The Australian National University, Poster Day, Tech. Rep., July 2010. [Online]. Available: http://cecs.anu.edu.au/files/posters10/47737500000000139.pdf

[7] T. Courtade, B. Xie, and R. Wesel, "Optimal exchange of packets for universal recovery in broadcast networks," in Proc. IEEE Military Communications Conference (MILCOM), San Jose, CA, Nov. 2010.

[8] D. Ozgul and A. Sprinston, "An algorithm for cooperative data exchange with cost criterion," in Information Theory and Applications Workshop (ITA), San Diego, CA, Feb. 2011.

[9] S. Y. R. Li, R. W. Yeung, and N. Cai, "Linear network coding," IEEE Trans. Inf. Theory, vol. 49, pp. 371-381, Feb. 2003.

[10] M. Bakshi and M. Effros, "On achievable rates for multicast in the presence of side information," in Proc. IEEE Int. Symp. Inf. Theory (ISIT), Toronto, ON, July. 2008, pp. $1661-1665$.

[11] R. W. Yeung and Z. Zhang, "Distributed source coding for satellite communications," IEEE Trans. Inf. Theory, vol. 45, pp. 1111-1120, May 1999.

[12] D. Lun, N. Ratnakar, M. Medard, R. Koetter, D. Karger, T. Ho, E. Ahmed, and F. Zhao, "Minimum-cost multicast over coded packet networks," IEEE/ACM Transactions on Networking, vol. 14, pp. 26082623, June 2006

[13] K. Jamieson and H. Balakrishnan, "PPR: Partial packet recovery for wireless networks," in ACM SIGCOMM, Kyoto, Japan, Oct. 2007.

[14] R. K. Jain, D. W. Chiu, and W. R. Hawe, "A quantitative measure of fairness and discrimination for resource allocation in shared computer system,” DEC-TR-301, Tech. Rep., 1984.

[15] O. L. Mangasarian, "Uniqueness of solution in linear programming," Linear Algebra and its Applications, vol. 25, pp. 151-162, June 1979.

[16] D. P. Bertsekas, Nonlinear Programming. Nashua, NH: Athena Scientific, 1999. 